02

\title{
Моделирование сигналов комбинационного рассеяния в биологических тканях прямым и двухшаговым подходами
}

\author{
(C) И.В. Красников ${ }^{1}$, А.Ю. Сетейкин ${ }^{1}$, B. Roth ${ }^{2}$, M. Meinhardt-Wollweber ${ }^{2}$ \\ ${ }^{1}$ Амурский государственный университет, \\ 675027 Благовещенск, Россия \\ ${ }^{2}$ Hannover Centre for Optical Technologies, \\ 30167 Hannover, Germany \\ e-mail: ivkrasnikov@gmail.com
}

Поступила в редакцию 23.08.2017 г.

\begin{abstract}
Проведен поиск эффективного метода моделирования задачи спектроскопии комбинационного рассеяния в мутных (рассеивающих) средах с учетом соответствующих параметров детектора и объема выборки. В основе предложенной модели лежит решение уравнения переноса излучения методом Монте-Карло. Рассмотрены два принципиальных подхода к численному моделированию комбинационного рассеяния: прямая задача транспорта релеевского и рамановского фотонов в каждой точке среды и двухшаговая модель, в которой на первом шаге рассчитывается поток фотонов в среде с последующей генерацией соответствующего количества рамановских фотонов в каждой точке.
\end{abstract}

DOI: $10.21883 /$ OS.2018.02.45521.184-17

\section{Введение}

Конфокальную флуоресцентную микроскопию можно рассматривать как разновидность традиционной флуоресцентной микроскопии, которая позволяет исследовать внутреннюю микроструктуру клеток, причем не только фиксированных, но и живых, идентифицировать микроорганизмы, структуры клетки и отдельные молекулы, наблюдать динамические процессы в клетках. Конфокальная флуоресцентная микроскопия в дополнение к этому обеспечила возможность трехмерного субмикронного разрешения объекта и существенно расширила возможность неразрушающего анализа прозрачных образцов.

Спектроскопия комбинационного рассеяния (СКР) позволяет регистрировать неупруго рассеянные фотоны в среде. Эти фотоны имеют сдвиг в длине волны относительно возбуждающего их излучения. Разница энергии для каждого неупруго рассеянного фотона соответствует молекулярной структуре определенного исследуемого образца [1]. Таким образом, колебания молекул каждого из компонент производят характерный спектр комбинационного рассеяния или своеобразные „отпечатки пальцев“, которые могут быть использованы для определения химического и структурного состава образца [2]. Благодаря этой особенности спектроскопия комбинационного рассеяния света хорошо известна в хемометрике. Кроме того, поскольку СКР позволяет неинвазивным способом идентифицировать молекулы и даже молекулярные конфигурации (изоформы и т. д.) in situ, она все чаще применяется в самых разнообразных сферах деятельности. Область ее применения в биомедицинских исследованиях широка и включает в себя диагностику рака [3-7], атеросклероза [8] и экспресс-анализ крови [9].
Основные преимущества СКР света по сравнению с методами, основанными на флуоресценции, заключаются в том, что комбинационные сигналы являются более четкими и спектрально узкими, что улучшает разделение сигналов [2]. Помимо качественного анализа СКР теоретически может быть также использована для количественного определения числа активных в комбинационном рассеянии молекул, поскольку сигнал комбинационного рассеяния (КР) масштабируется с их концентрацией. На практике использование этого ценного свойства затрудняется ввиду быстрого затухания сигнала КР в мутной среде. На сегодняшний день задача надежного количественного определения величины затухания сигнала КР не может быть решена удовлетворительно из-за отсутствия адекватных моделей и надежных инструментов моделирования, с одной стороны, и недостаточного знания о влиянии оптических свойств и распределения рассеивателей в образце, с другой.

В настоящей работе были реализованы два различных подхода для теоретического моделирования КР моделирования с целью сравнения их эффективности и определения оптимальных режимов параметров для работы. В обоих случаях для моделирования сигнала комбинационного рассеяния применен метод МонтеКарло (МК). Мы использовали алгоритмы, основанные на хорошо известной реализации метода МК для рассеяния фотонов в мутных средах, разработанной Wang с соавт. [10], с минимизацией времени расчета по технологии NVidia CUDA. Следует заметить, что в работе имеются в виду не отдельные фотоны, а пакеты фотонов, т.е. группы нескольких фотонов, имеющих схожую траекторию движения.

Первый подход, „прямой метод“, основан на принципах моделирования флуоресценции, при этом распространение фотонов возбуждения моделируется класси- 
ческим алгоритмом Монте-Карло. После вычисления количества элементарных актов поглощения и рассеяния для данного пакета фотонов генерируется случайное число $\xi>P_{\text {Raman }}$ (где $P_{\text {Raman }}-$ это вероятность того, что на данном этапе произойдет КР), начальный пакет фотонов уничтожается и продолжается вычисление для комбинационно рассеянного фотона с новым направлением и длиной волны. Длина волны генерируемого фотона выбирается из диапазона возможных длин волн КР света с заданным распределением вероятностей, соответственно выбранному значению меняются оптические свойства среды. Каждому пакету фотонов падающего излучения присваивается статистический вес, равный единице, который уменьшается с каждым шагом за счет поглощения части фотонов в пакете. Начальный статистический вес сгенерированного КР фотона равен оставшемуся статистическому весу исходного фотона в момент, когда последний уничтожается.

Второй подход - двухступенчатый, также основанный на методе Монте-Карло, дает достаточно точные результаты моделирования. Этот метод не так интуитивно понятен, как „прямой“ метод, так как КР фотоны генерируются не при одиночных событиях, а в результате обхода каждого узла сетки с учетом статистического распределения всех фотонов возбуждения.

\section{1. Описание модели}

Различные подходы для моделирования распространения оптического излучения в сильно рассеивающих случайно-неоднородных средах, были широко освещены в многочисленных исследованиях [1,3-5]. Модели распространения оптического излучения в многокомпонентной случайно неоднородной рассеивающей среде могут быть разделены на две основные категории: стохастические вычислительные модели, такие как метод случайного блуждания и метод Монте-Карло, а также детерминированные модели, основанные на дифференциальных уравнениях в частных производных для плотности фотонов (электромагнитная теория, включая теорию Ми и рассеяние Релея-Ганса, или теория переноса излучения). И стохастические, и детерминированные модели направлены на выяснение оптимальной конструкции оптической системы и интерпретации результатов измерений. Кроме того, необходимо принять две основные концепции модели биологической ткани: оптические свойства биоткани имеют непрерывное (случайное) распределение по всему объему и ткань представляется совокупностью дискретных рассеивателей и поглотителей [3].

Большинство существующих моделей КР используют двухступенчатую модель, когда рассчитывается распределение падающего излучения по классической схеме, а затем, на основе этого распределения, в каждом узле расчетной сетки производится повторный запуск фотонов с новой длиной волны и случайно выбранным направлением [10-13]. Основанием для такого подхода является тот факт, что КР приблизительно на 10 порядков слабее флуоресценции. Для усовершенствованных методов КР, таких как резонансное КР, сечение рассеяния на 5 порядков выше, но все же число моделируемых фотонов крайне мало. Тем не менее возможность моделирования релеевского и комбинационного рассеяния за один проход показали Everall и др. в своей работе [14], где применили МК для моделирования КР и фотонов рассеяния Тиндаля в непрозрачных средах при различных условиях. Чтобы получить достаточное число комбинационных фотонов, приходится использовать неправдоподобно большое значение для вероятности комбинационного рассеяния $\left(P_{\text {Raman }}=0.01\right)$. Установлено, что получаемая кинетика затухания КР не зависит от величины вероятности КР, по крайней мере до выбранных значений [14]. В процессе реализации стало очевидно, что данный подход является очень затратным, поэтому существует немного работ, посвященных моделированию фотонов КР света прямым пересчетом.

Метод Монте-Карло для слабых процессов обычно не подходит, так как малое количество конечных фотонов приводит к ненадежным статистическим выводам в результате моделирования. Чтобы справиться с этой проблемой, оба представленных в настоящей работе подхода используют некоторые допущения, позволяющие получить достаточное количество пакетов фотонов: во-первых, это запуск КР фотонов после предварительного расчета распределения поглощенной энергии в среде в двухступенчатом подходе и использование вероятности КР, значительно превышающей реальную величину в случае прямого подхода. Следует отметить, что и прямой, и двухступенчатый подходы используют методы и алгоритмы, аналогичные методу МК рассеяния фотонов в мутных средах, описанных Wang и др. в работе $[10]$, и его интерпретации для параллельного вычисления.

\section{1. Прямой подход}

Предполагается, что коэффициент поглощения $\mu_{a}\left(\lambda_{\text {Raman }}\right)$ и коэффициент для $\mu_{s}\left(\lambda_{\text {Raman }}\right)$ КР постоянны по всему образцу. Также используется предположение, что фотоны КР могут быть получены в любой точке вдоль траектории фотона падающего светового пучка с вероятностью $P_{\text {Raman }}$, которая постоянна для каждого шага. При моделировании на каждом шагу $t$ выбирается случайное число $\xi$, равномерно распределенное между 0 и 1 . Если $\xi$ попадает между 0 и $P_{\text {Raman, }}$,о создается фотон КР, который помещается для удобства в текущую точку положения родительского фотона (рис. 1). Направление движения фотона КР на первом шаге задается случайным образом, что соответствует изотропному молекулярному рассеянию. Для распространения КР фотонов учитываются оптические свойства среды, соответствующие длине волны КР. В свою очередь длина волны выбирается случайным образом из спектра КР 


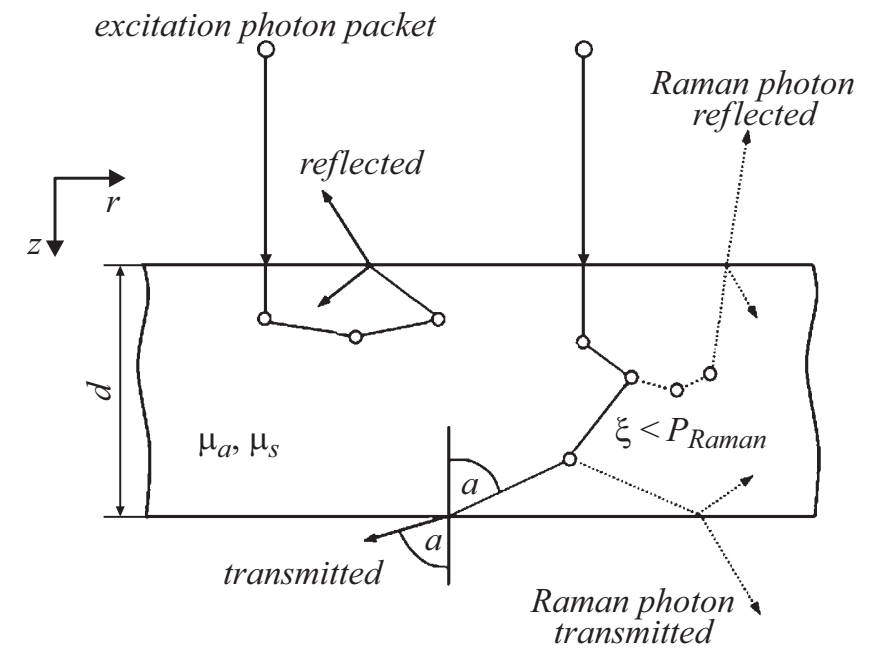

Рис. 1. Общая схема модели МК для КР. На каждом шаге (кружки) часть фотонов из пакета фотонов поглощается. Сплошные линии показывают траектории упруго рассеянных фотонов, траектории комбинационно рассеянных фотонов представлены в виде пунктирных линий. Глубина среды $z, r-$ радиальная координата.

для данной среды. Чтобы получить достаточное число комбинационных фотонов за один проход, используется большое значение $P_{\text {Raman }}=0.01$. В реальности, величина $P_{\text {Raman }}$ на 5-10 порядков меньше вероятности релеевского рассеяния, тем не менее в соответствии с работой Everall и др. [14] такое предположение вполне оправдано.

Следует обратить внимание, что в то время как в прямом методе фотоны КР генерируются случайным образом из родительских фотонов, в двухступенчатом методе эти фотоны генерируются из распределения плотности поглощенной энергии падающего излучения.

\section{2. Двухступенчатый подход}

Двухступенчатый метод создает достаточное количество рассеянных фотонов без увеличения величины $P_{\text {Raman }}$ или числа исходных фотонов. Первый шаг идентичен алгоритму МК, описанному в работе [10], он моделирует распространение падающего излучения через образец $A_{e x}(r, z)$. В сочетании с коэффициентом поглощения $\mu_{a}$ это приводит к распределению фотонов возбуждения в образце $\Phi_{e x}(r, z)$. На втором этапе изотропно запускаются рамановские фотоны в каждой точке среды с весом, равным

$$
W_{\text {Raman }}=\Phi_{e x}(r, z) \cdot \mu_{\text {Raman }},
$$

где $r$ - радиальная координата в изотропном случае. Коэффициент $\mu_{\text {Raman }}$ сравним с вероятностью $P_{\text {Raman }}$ для прямого метода. Если только один фотон в каждой единице объема образца был создан, это не привело бы к достаточно большому числу фотонов, необходимых для получения хороших статистических результатов моделирования. Таким образом, этот алгоритм создает любое желаемое число фотонов в образце. Распределение фотонов, начиная со второй стадии, аналогично распределению фотонов возбуждения $\Phi_{e x}(r, z)$. Другими словами, мы масштабируем $\Phi_{e x}(r, z)$ к числу фотонов, которые мы намерены выделить на сетке моделирования, которое определяется $A_{e x}(r, z)$.

Комбинационно рассеянные фотоны имеют соответствующий сдвиг в длине волны. Выбор новой длины волны задается спектром комбинационного рассеяния. В данной работе требуемый спектр комбинационного рассеяния был получен экспериментально, но в целом заранее рассчитанные спектры комбинационного рассеяния также могут быть использованы в качестве исходных данных. Как предполагается, коэффициент рассеяния очень мал $\left(\mu_{\text {Raman }}<10^{-6} \mathrm{~cm}^{-1}\right)$, множественным КР можно пренебречь и, таким образом, выбранная длина волны у фотона не меняется. После того, как фотон запущен, он движется через среду так же, как исходные фотоны возбуждения на первом этапе, но с другими оптическими характеристиками среды, соответствующими новой длине волны.

\section{2. Результаты и обсуждение}

Чтобы сравнить достоинства и недостатки обоих подходов, были использованы данные из литературных источников и проведенных экспериментов. Моделировалось воздействие лазерного пучка с длиной волны $488 \mathrm{~nm}$, с гауссовым профилем и диаметром $100 \mu \mathrm{m}$, падающего ортогонально. В качестве объекта исследования КР был выбран раствор бета-каротина в этаноле. Коэффициент поглощения для $0.01 \mathrm{mg} / \mathrm{l}$ бета-каротина взят из пакета PhotochemCAD и масштабирован в соответствии с данными из [15] (при $452 \mathrm{~nm})$. В результате получено значение $\mu_{a}=4.818 \mathrm{~cm}^{-1}$ при длине волны $488 \mathrm{~nm}$, показатель преломления раствора $n=1.366$ (этанол), $\mu_{s}=944.0 \mathrm{~cm}^{-1}$ (этанол), а коэффициент анизотропии для прозрачной среды принят $g=0.97$. Полученные в результате моделирования параметры распределения света в образце, такие как диффузное отражение (число фотонов, покидающих объем рассеяния вверх), коэффициент пропускания (число фотонов, покидающих объем рассеяния в направлении сверху вниз) и поглощения, были выбраны в качестве выходных данных для сравнения и оценки результатов моделирования.

На кювету с раствором бета-каротина воздействует лазерное излучение конфокального микроскопа. Падающий пучок сфокусирован на заданной глубине, в нашем случае это 100, 200 и $300 \mu \mathrm{m}$. Меньшая глубина не имеет практического смысла, а на большей глубине величина интенсивности сигнала КР значительно затухает.

Исследуемый объем представляет собой усеченный конус, заключенный между сечением падающего пучка поверхностью среды и минимальным сечением пучка в фокальной плоскости. Из геометрической оптики радиус 


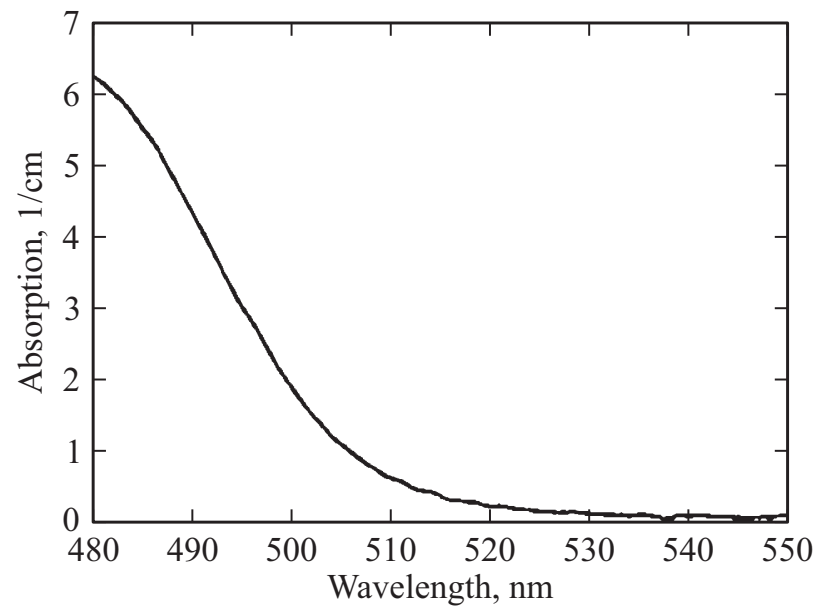

Рис. 2. Коэффициент поглощения бета-каротина как функция от длины волны.

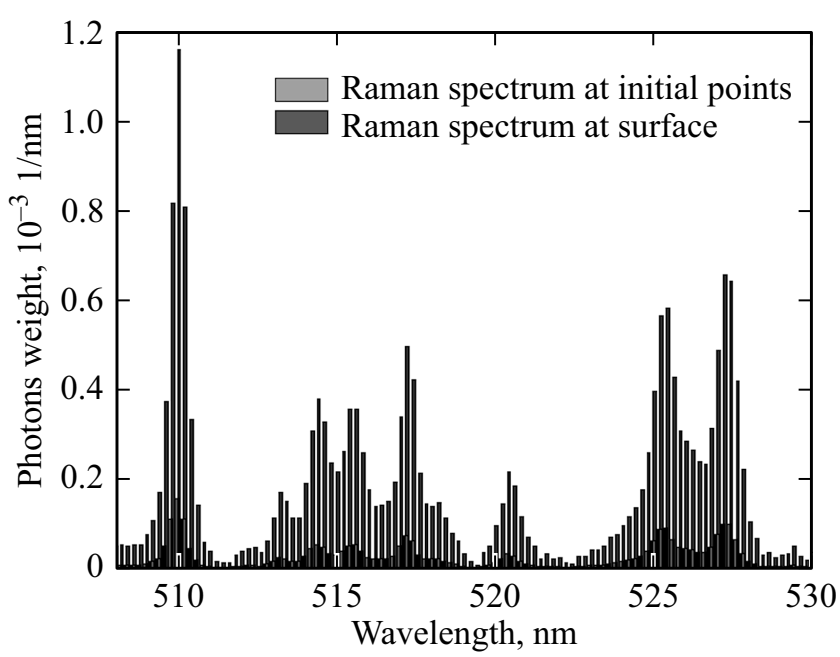

Рис. 3. Моделирование спектра комбинационного рассеяния в растворе бета-каротина в этаноле на детекторе (черный), исходный спектр - серый. Длина волны возбуждающего излучения $488 \mathrm{~nm}$.

основания этого конуса может быть выражен как

$$
R(d, z)=r_{0}+(d-z) \cdot \tan \left(\arcsin \left(\frac{N A}{n 2}\right)\right),
$$

где $d-$ глубина фокальной плоскости, $r_{0}-$ радиус изображения пятна в фокальной плоскости, и $n-$ показатель преломления.

Для параметров эксперимента радиус фокального пятна $\left(r_{0}\right)$ может быть вычислен:

$$
r_{0}=\frac{n 2}{n 1} \frac{\lambda}{\pi \cdot N A}=\frac{1.366}{1.0} \cdot \frac{0.532}{\pi}=0.2313 \mu \mathrm{m} .
$$

Для глубины фокуса $d=100$ мкм радиус пучка на поверхности составит $R=108 \mu \mathrm{m}$, для $d=200 \mu \mathrm{m}$ $R=215 \mu \mathrm{m}$, и для $d=300 \mu \mathrm{m} R=323 \mu \mathrm{m}$ соответственно.
Разработанный алгоритм использует заданные оптические параметры в зависимости от длины волны конкретного пакета фотонов. Так как комбинационно рассеянные фотоны имеют отличную от падающих фотонов длину волны, программа применяет оптические параметры среды для соответствующей длины волны фотонов КР света, пока новый рассеянный фотон „полностью“ поглощается или выходит из образца. Это важный аспект из-за сильного поглощения бета-каротина в диапазоне длины волны возбуждения.

Так как мы рассматриваем резонансные комбинационные процессы, возбуждающая длина волны находится рядом с пиком поглощения бета-каротина (рис. 2). Таким образом, ослабление сигнала значительно меняется в спектре КР.

Каждому новому КР фотону задается соответствующая длина волны (рис. 3). Длина волны определяется функцией распределения вероятности, полученной из экспериментально определенного спектра КР [15]. В обоих методах моделирования мы изучаем и сравниваем два класса фотонов: упруго рассеянные (релеевские) фотоны и неупруго рассеянные фотоны (КР).

В ходе эксперимента фокус пучка движется вдоль оси $z$, а детектор фиксирует уровень сигнала, что
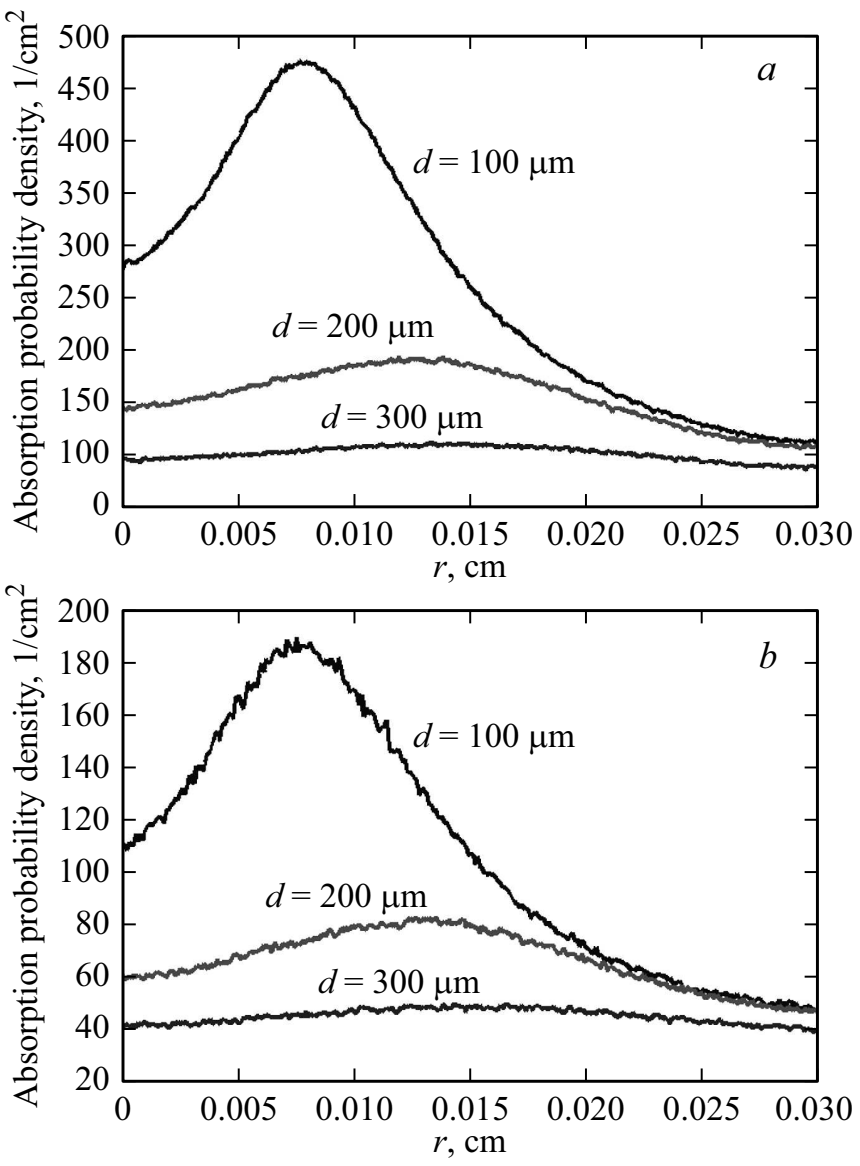

Рис. 4. Распределение плотности вероятности поглощения КР фотонов вдоль оси $z$ при моделированиии прямым $(a)$ и двухступенчатым $(b)$ методами. 

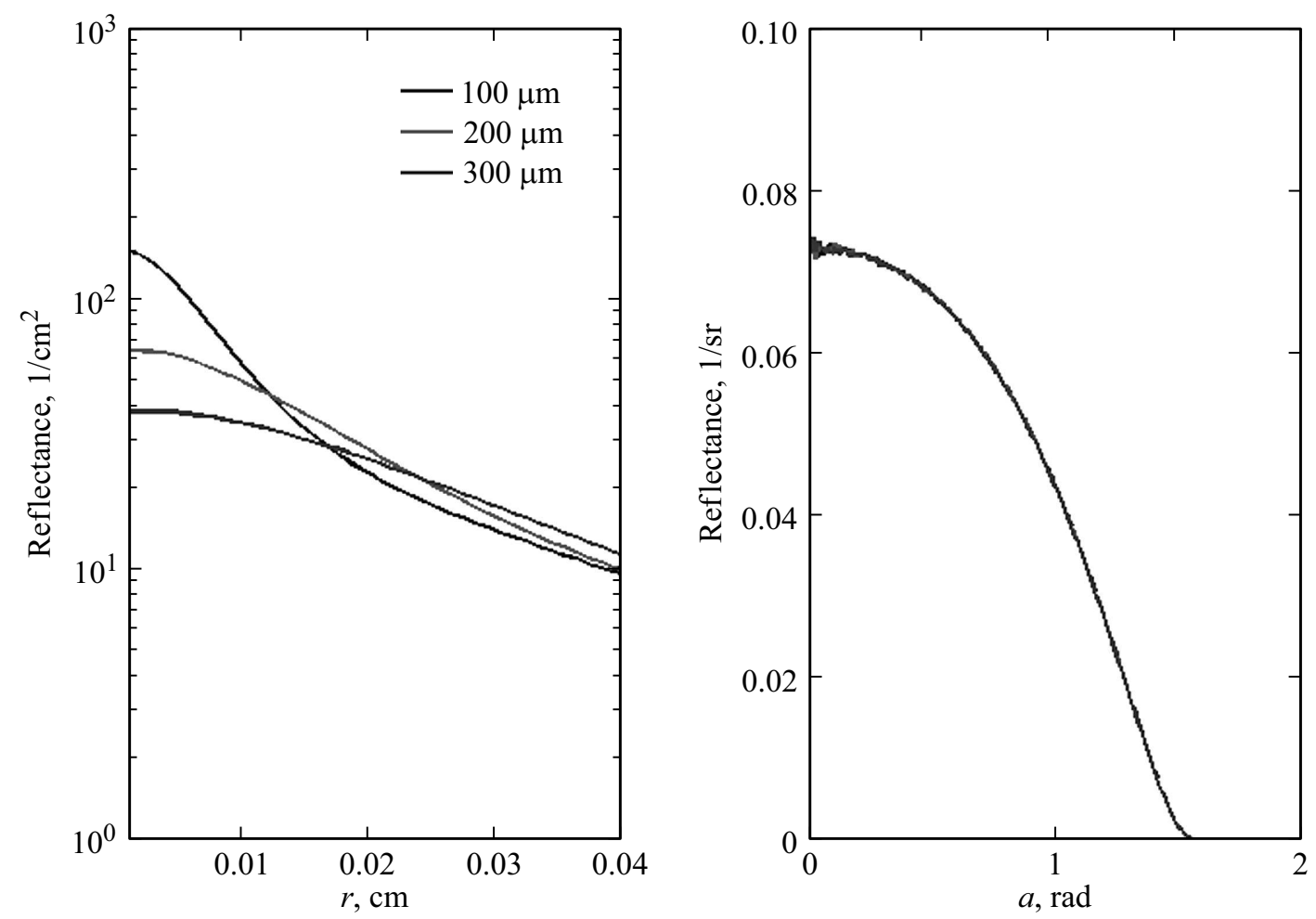

$a$
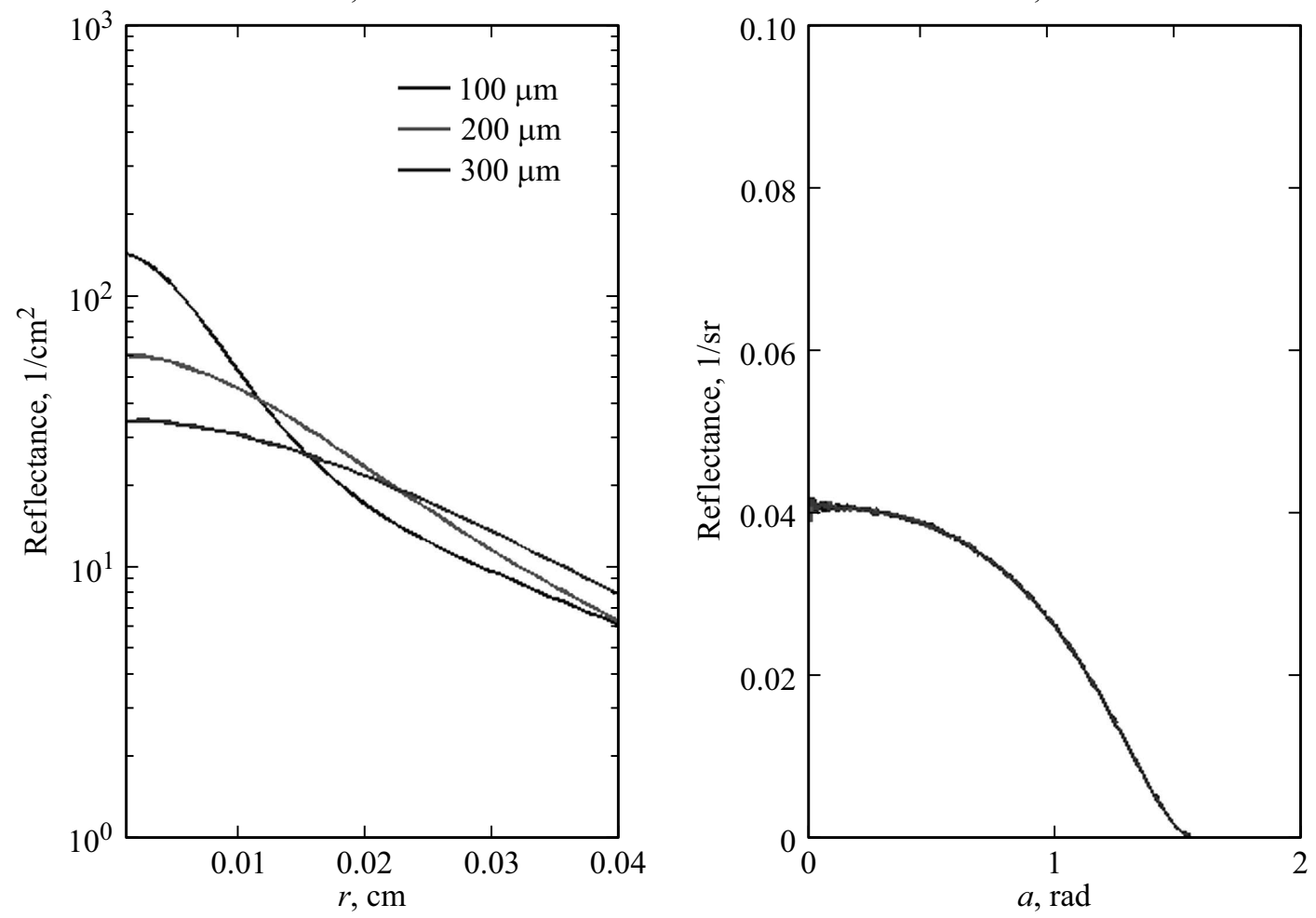

Рис. 5. Сравнение величины полного отражения изучения на поверхности (на детекторе) при моделированиии прямым (a) и двухступенчатым (b) методами.

позволяет судить о внутренней морфологии, составе и структуре исследуемого образца. В численном эксперименте используется глубина 100, 200 и $300 \mu \mathrm{m}$. Величина плотности вероятности поглощения фотона вдоль оси $z$ для разной глубины фокуса представлена на рис. 4. Видно, что независимо от выбранного метода профиль кривых совпадает, что говорит о достоверности полученных данных.

Уровень сигнала (полное отражение фотонов) на моделируемом детекторе представлен на рис. 5. Видно, 
Таблица 1. Зависимость доли полученных на детекторе КР фотонов, относительно полного отражения КР фотонов образца, как функция угла детектора

\begin{tabular}{c|c|c|c|c}
\hline Фокусная глубина & $10^{\circ}$ & $30^{\circ}$ & $45^{\circ}$ & $60^{\circ}$ \\
\hline$z<100 \mu \mathrm{m}$ & $63.3 \%$ & $85.9 \%$ & $92.6 \%$ & $96.5 \%$ \\
$z<200 \mu \mathrm{m}$ & $42.7 \%$ & $79.5 \%$ & $89.4 \%$ & $95.0 \%$ \\
$z<300 \mu \mathrm{m}$ & $31.5 \%$ & $72.2 \%$ & $86.1 \%$ & $93.5 \%$
\end{tabular}

Таблица 2. Диффузное отражение, поглощенная и прошедшая среду часть комбинационно рассеянного излучения, $P_{\text {Raman }}=0.01, n=1.366, \mu_{a}=4.818 \mathrm{~cm}^{-1}, \mu_{s}=944.0 \mathrm{~cm}^{-1}$, $g=1$

\begin{tabular}{l|c|c}
\hline \multicolumn{1}{c|}{$\begin{array}{c}\text { Оптические } \\
\text { характеристики }\end{array}$} & $\begin{array}{c}\text { Двухступенчатый } \\
\text { метод }\end{array}$ & \multicolumn{1}{c}{$\begin{array}{c}\text { Прямой } \\
\text { метод }\end{array}$} \\
\hline \multicolumn{3}{c}{ Релеевское рассеяние } \\
\hline Зеркальное отражение & 0.0239295 & 0.0239295 \\
Диффузное отражение & 0.304982 & 0.125863 \\
Поглощенная часть & 0.191490 & 0.0957153 \\
Прошедшая часть & 0.479574 & 0.245337 \\
\hline \multicolumn{3}{c}{ Комбинационное рассеяние } \\
\hline Диффузное отражение & 0.136331 & 0.229381 \\
Поглощенная часть & 0.034566 & 0.034594 \\
Прошедшая часть & 0.147135 & 0.245002
\end{tabular}

что значительные различия в профиле сигнала с различной глубины находятся в узком круге диаметром не более $0.05 \mathrm{~cm}$. Распределения уровня сигнала по углам рассеяния совпадают.

\section{Заключение}

Из представленных результатов можно сделать вывод, что оба используемых в работе подхода к моделированию распространения КР излучения в мутных средах дают хорошие результаты. На рисунках видно, что конечные величины плотности вероятности поглощения различаются, однако следует учитывать, что эти величины не связаны с физическими свойствами среды и эксперимента. Количество запущенных КР фотонов обусловливается искусственно введенной вероятностью КР, а в случае двухступенчатого метода также искусственно задается число фотонов, генерируемых в точках сетки.

В табл. 2 приведено сравнение результатов моделирования. Видно, оба подхода дают равные части комбинационно рассеянных фотонов.

Модель реализована с использованием технологии NVidia CUDA для увеличения скорости вычислений и оптимизирована для получения высокоточных результатов. Для двухэтапного метода вычисления, выполненные на ноутбуке с графической картой NVidia GeForce GT 750M, занимают около $130 \mathrm{~s}$. Для прямого метода длительность расчета составляет 40 или $300 \mathrm{~s}$ при использовании $10^{8}$ или $10^{9}$ фотонов соответственно.

Анализ полученных результатов приводит к выводу, что оба метода имеют преимущества в разных режимах параметров. Двухшаговый метод обычно приводит к плавному профилю кривых, поскольку запуск большого количества рамановских рассеянных фотонов на втором этапе обеспечивает хорошее статистическое представление случайных событий. Реализация двухшагового метода более понятна и проста и не требует серьезной модификации исходного кода Монте-Карло. Прямой метод имеет то преимущество, что его процедура ближе к реальным процессам в среде, облучаемой лазером. Здесь хорошая статистика фотонов может быть достигнута только для $P_{\text {Raman }} \geq 0.01$, но в рамках этого ограничения она позволяет моделировать процессы с хорошей точностью за приемлемое время.

В дальнейшей работе планируется учитывать распределение свойств рассеяния в среде и параметры детектора. При том, что оптические параметры реальных образцов известны, часто бывает трудно получить точные и подробные параметры оптических свойств среды для полного описания эксперимента. Так, очень важно экспериментально определить, как молекулы бетакаротина распределяются в растворе, независимо от того, образуют ли они агрегаты между собой или с другими молекулами и есть ли фотореакция, вызванная лазерным излучением. Поэтому на следующем этапе мы планируем провести эксперимент для измерения профилей КР для разных растворов с четко определенными размерами частиц (например, полимерных сфер, чернил) и сравнить их с имитируемыми профилями.

Работа выполнена по госзаданию 3.9994.2017/5.2 Проведение научно-исследовательских работ в рамках международного научно-образовательного сотрудничества по программе „Михаил Ломоносов“.

\section{Список литературы}

[1] Matousek P., Draper E.R.C., Goodship A.E., Clark I.P., Ronayne K.L., Parker A.W. // Appl. Spectrosc. 2006. V. 60. P. 758-763.

[2] Shih W.-C., Bechtel K.L., Feld M.S. // Opt Express., 2008. V. 16. N 17. P. $12726-12736$.

[3] Tuchin V.V. Handbook of Optical Biomedical Diagnostics, Washington: SPIE Press, 2002, p. 84.

[4] Meglinski I., Doronin A.V. // Advanced Biophotonics: tissue optical sectioning / Ed. by Tuchin V.V., Wang R.K. Taylor \& Francis, 2012.

[5] Zhu C., Liu Q. // J. Biomed. Optics. 2013. V. 18. N 5. P. 050902.

[6] Harmsen S., Huang R., Wall M.A., Karabeber H., Samii J.M., Spaliviero M., White J.R., Monette S., O'Connor R., Pitter K.L., Sastra S.A., Saborowski M., Holland E.C., Singer S., Olive K.P., Lowe S.W., Blasberg R.G., Kircher M.F. // Science Translational Medicine. 2015. V. 7. N 271. P. 271-278. 
[7] Nijssen A., Koljenovic S., Bakker Schut T.C., Caspers P.J., Puppels G.J. // J. Biophotonics, 2009. V. 2. P. 29-36.

[8] Marzec K., Wrobel T., Rygula A., Maslak E., Jasztal A., Fedorowicz A., Chlopicki S., Baranska M. // J. Biophotonics. 2014. V. 7. N 9. P. 744-756.

[9] Berger A.J., Koo T.-W., Itzkan I., Horowitz G., Feld M.S. // App. Optics. 1999. V. 38. N 13. P. 2916-2926.

[10] Wang L.H., Jacques S.L., Zheng L.Q. // Comput. Methods Programs Biomed. 1995. V. 47. P. 131-146.

[11] Welch A.J., Gardner C., Richards-Kortum R., Chan E., Criswell G., Pfefer J., Warren S. // Lasers Surg. Med. 1997. V. 21. N 2. P. $166-178$.

[12] Wang S., Zhao J., Lui H., He Q., Bai J., Zeng H. // J. Biophotonics. 2014. V. 7. N 9. P. 703-715.

[13] Reble C., Gersonde I., Andree S., Eichler H.J., Helfmann J. // J. Biomed. Opt. 2010. V. 15. N 3. P. 037016-1-037016-8.

[14] Everall N., Hahn T., Matousek P., Parker A.W., Towrie M. // Appl Spectrosc. 2004. V. 58. N 5. P. 591-7.

[15] Zechmeister L., Polgar A. // J. Am. Chem. Soc. 1943. V. 65. P. $1522-1528$. 